\title{
Embeddable Advanced Sensors for Harsh Environment Sensing Applications
}

\author{
Lei Yuan, ${ }^{1}$ Yinan Zhang, ${ }^{2}$ Yan Lu, ${ }^{3}$ Xiaobei Zhang, ${ }^{4}$ and Mengmeng Wang \\ ${ }^{1}$ Department of Electrical and Computer Engineering, Clemson University, Clemson, SC 29634, USA \\ ${ }^{2}$ Department of Electrical and Computer Engineering, Missouri University of Science and Technology, Rolla, MO 65409, USA \\ ${ }^{3}$ Research Center of Nondestructive Testing \& Evaluation, Beijing University of Technology, Beijing 100124, China \\ ${ }^{4}$ School of Communication and Information Engineering, Shanghai University, Shanghai 200072, China \\ ${ }^{5}$ Department of Electrical Engineering, University of Nebraska-Lincoln, Lincoln, NE 68588-0511, USA
}

Correspondence should be addressed to Lei Yuan; yuan7@g.clemson.edu

Received 4 December 2016; Accepted 4 December 2016

Copyright (c) 2016 Lei Yuan et al. This is an open access article distributed under the Creative Commons Attribution License, which permits unrestricted use, distribution, and reproduction in any medium, provided the original work is properly cited.

Research and development in advanced sensors with embedded monitoring capability have experienced significant growth in recent years, fueled by their broad applications in real-time measurement of a wide variety of physical, chemical, and biological quantities. Compared with conventional sensors with bulky assemblies, recent progress in 3D manufacturing technologies (e.g., ultrafast laser micromachining and additive manufacturing) has opened up a new avenue in one-step fabrication of assembly-free micro devices in various materials as well as the development of compact, customized, and intricate smart structures/components. The merits of these advanced manufacturing techniques enable the integration of embeddable advanced sensors into smart structures and components for improved robustness, enriched functionality, enhanced intelligence, and unprecedented performance.

This special issue seeks to attract researchers to contribute their original research articles as well as review articles that will further improve the performance of advanced sensors. Particular focus is placed on smart parts (advanced sensors and smart structures/components) to address the monitoring needs within extreme environment conditions such as high temperature, high pressure, corrosive/erosive atmosphere, and large strain/stress. A well-chosen real practical application example, based on novel methods and/or known methods to new fields, will also be considered. Both experimental and theoretical/simulated results are welcome to this special issue.
The special issue consists of 12 papers whose brief summaries are listed below.

"Compressed Sensing, Pseudodictionary-Based, Superresolution Reconstruction" by $\mathrm{C}$. Li et al. focuses on using the classical OMP reconstruction algorithm to solve the sparse optimization problem and effectively increase image resolution. The proposed method provides an effective way to improve the application range and accuracy of aviation and aerospace photogrammetric images.

"WildSense: Monitoring Interactions among Wild Deer in Harsh Outdoor Environments Using a Delay-Tolerant WSN" by J. Ahn et al. developed, deployed, and evaluated a new system for monitoring the movement patterns and interaction behaviors of free-range deer in a rugged wilderness environment. The system combines the functionalities of both GPS and RF-radio sensors with low-cost and minimalresource motes. It is able to operate robustly for a period of up to several months for continual tracking and monitoring of the locations and interaction behaviors of wild deer in harsh environments.

"Location Fingerprint Extraction for Magnetic Field Magnitude Based Indoor Positioning" by W. Shao et al. analyzes the main interference sources of the magnetometer embedded on smartphone and presents feature distinguishability measurement technique to evaluate the performance of different feature extraction methods. Experiments revealed that selected fingerprints will improve position distinguishability. 
"Measurement of Circumferential Liquid Film Based on LIF and Virtual Stereo Vision Sensor" by T. Xue et al. presents a measurement platform based on the laser-induced fluorescence (LIF) and virtual stereo vision sensor. The experimental results show that the method is valid and effective, which can give a more detailed characterization of the liquid film to reveal the flow structures and flow mechanism.

"Characteristics of Eddy Current Distribution in Carbon Fiber Reinforced Polymer" by S. Jiao et al. studies the characteristics of eddy current (EC) distribution in carbon fiber reinforced polymer (CFRP) laminates. The authors developed an electromagnetic field computation model of EC response to CFRP based on the finite element method. The simulation results are beneficial to optimizing sensor design and testing parameters, as well as damage detection and evaluation.

"Design and Fabrication of Air-Based 1-3 Piezoelectric Composite Transducer for Air-Coupled Ultrasonic Applications" by C. He et al. designed and fabricated air-based 1-3 piezoelectric composite transducers in order to solve the acoustic impedance matching problem. Simulation and experiments show that 3D printed air-based 1-3 piezoelectric composites structures are appropriate for fabricating the air-coupled transducers. They can both reduce the acoustic impedance and enhance the electromechanical conversion efficiency.

"New Leakage Current Particulate Matter Sensor for OnBoard Diagnostics" by J. Wang et al. introduced the structure and principle of a new leakage current particulate matter (PM) sensor and performed further study on the PM sensor with combination of numerical simulation and bench test. The results of simulation and experiment reveal the possibility of PM concentration (mass) detection by the sensor.

"An IFPI Temperature Sensor Fabricated in an Unstriped Optical Fiber with Self-Strain-Compensation Function" by Y. Song et al. describes an intrinsic Fabry-Perot interferometry (IFPI) temperature sensor with self-strain-compensation function. Experiment shows the proposed sensor structure holds a constant temperature sensitivity when strained differently.

"Research on Fused Tapered Photonic Crystal Fiber Sensor Based on the Method of Intermittent Cooling" by $\mathrm{G}$. Fu et al. proposed a fused tapered photonic crystal fiber interferometer based on the intermittent cooling method. The fringe contrast of the transmission spectra of this sensor is larger than ordinary fused tapered method. The sensor has high sensitivity to refractive index and low temperature crosssensitivity.

"Research on Application of Wax Deposition Detection in the Nonmetallic Pipeline Based on Electrical Capacitance Tomography" by N. Li et al. developed an ECT (electrical capacitance tomography) sensor for wax deposition detection in nonmetallic pipelines. The experimental results indicate that the ECT system is valid and feasible for detecting the degree of wax deposition in the nonmetallic pipelines.

"Development of a Wireless Temperature Sensor Using Polymer-Derived Ceramics" by R. Zhao et al. developed a temperature sensor using an embedded system and a sensor head made of polymer-derived SiAlCN ceramics (PDC). The developed temperature sensor has been experimentally tested to demonstrate the possibility of using such sensors for real world applications.

"Evaluation of the Degradation on a COTS Linear CCD Induced by Total Ionizing Dose Radiation Damage" by $\mathrm{Z}$. Wang et al. presented the evaluation of the degradation on a COTS linear Charge Coupled Device (CCD) induced by total ionizing dose (TID) radiation damage. The dark current, dark signal nonuniformity (DSNU), photo response nonuniformity (PRNU), saturation output, full well capacity (FWC), quantum efficiency (QE), and responsivity versus the TID were analyzed. The behavior of the tested CCD had shown remarkable degradation after radiation. The degradation mechanisms of the CCD induced by TID damage were also discussed.

\section{Acknowledgments}

Introducing this special issue to this journal, we would like to thank all the researchers for their contributions and reviewers for their help in achieving a high technical quality of papers in this special issue. The Lead Guest Editor Dr. Lei Yuan would like to thank all the Guest Editors for their valuable contribution to this special issue. We hope all the readers can enjoy the papers in the special issue as we do.

Lei Yuan

Yinan Zhang Yan $\mathrm{Lu}$

Xiaobei Zhang

Mengmeng Wang 


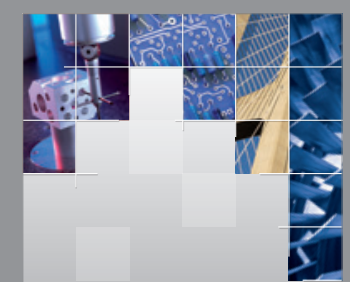

\section{Enfincering}
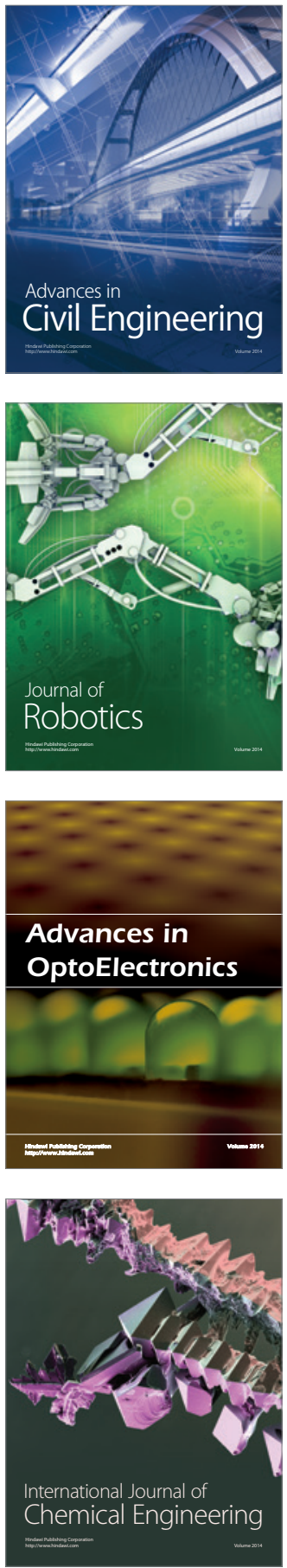

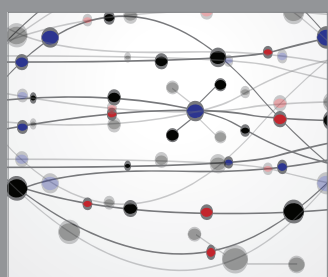

The Scientific World Journal

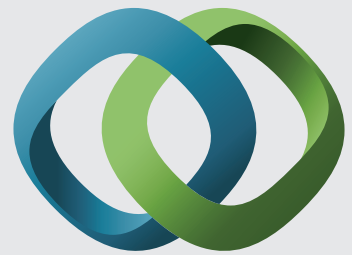

\section{Hindawi}

Submit your manuscripts at

http://www.hindawi.com
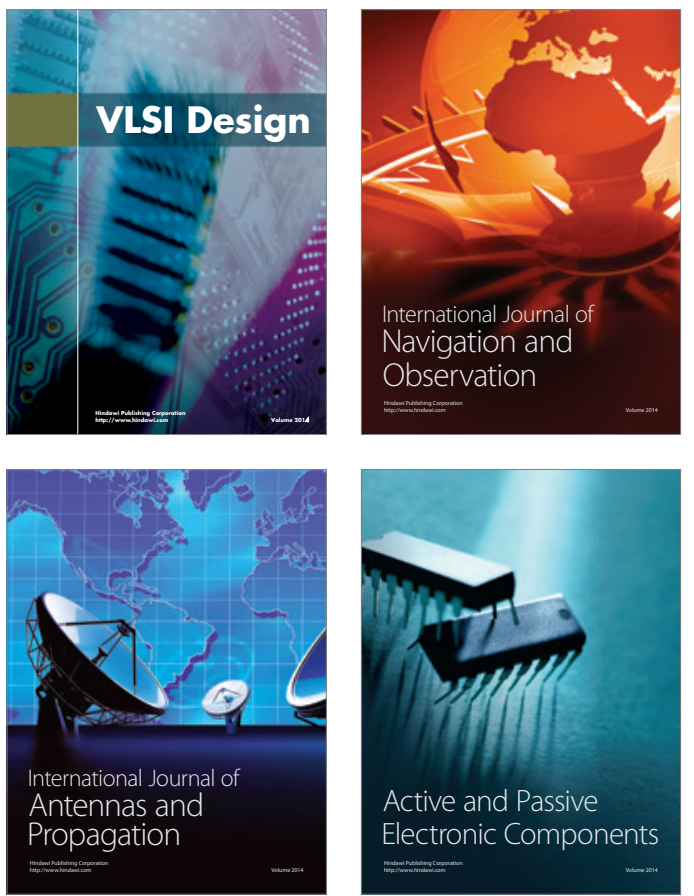
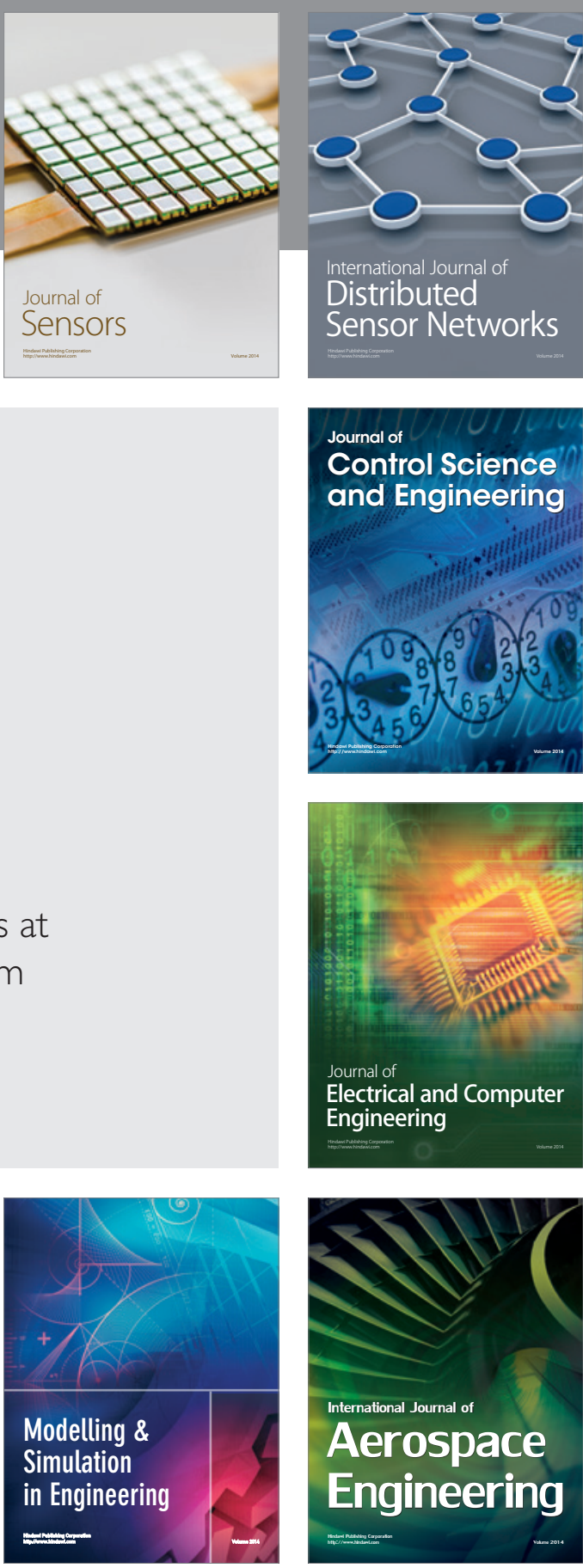

International Journal of

Distributed

Sensor Networks

Journal of

Control Science

and Engineering
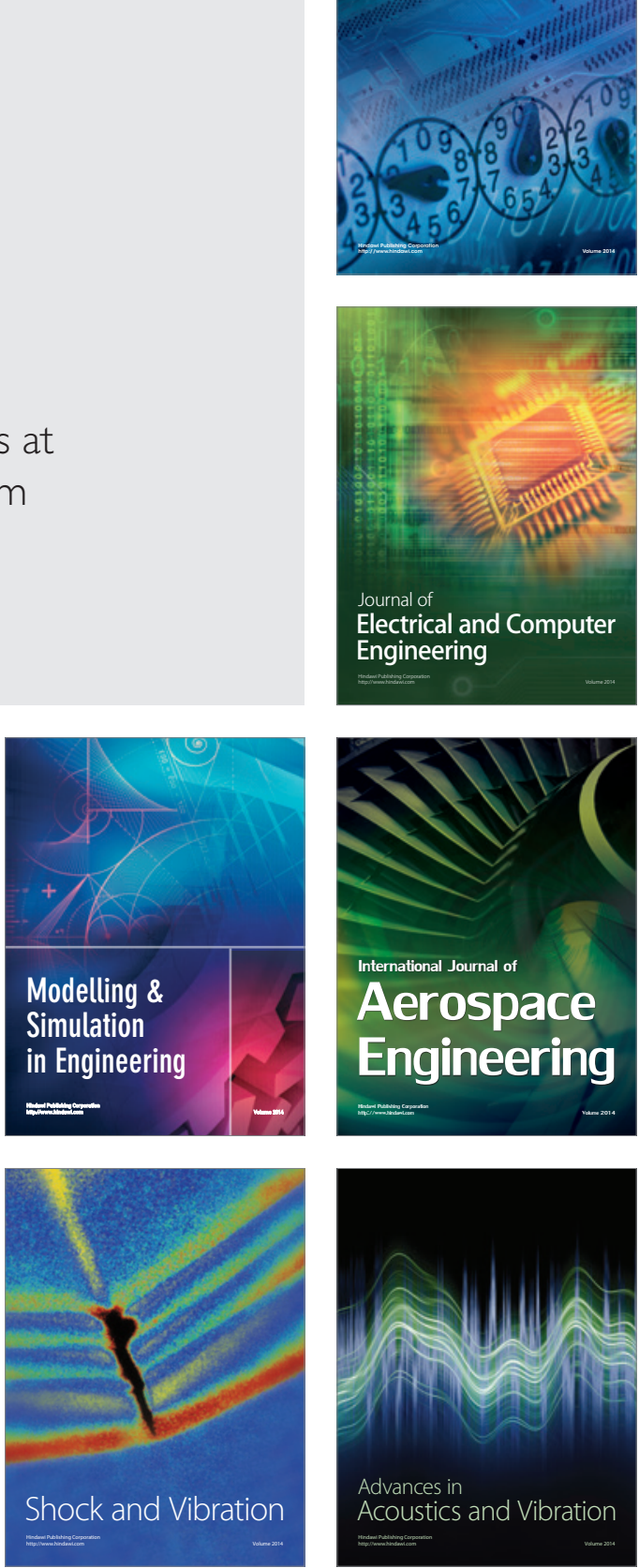